\title{
Speed Control for Reduced Risk of Collision with Oncoming Vehicles in Obstacle Avoidance Scenarios
}

\author{
A. Arikere \\ TTC - Trollhättan Technical Center \\ AAM (American Axle \& Manufacturing) Inc., Trollhättan, Sweden
}

M. Klomp

Vehicle Dynamics CAE

Volvo Car Corporation, Göteborg, Sweden

M. Lidberg

Department of Applied Mechanics

Chalmers University of Technology, Göteborg, Sweden

ABSTRACT: When a driver performs an evasive steering manoeuvre in order to avoid an obstacle, there is an increased risk of collisions with oncoming vehicles in the adjacent lane. A controller to reduce this risk of secondary collision by regulating the vehicle speed is designed and implemented. Simulations are preformed in IPG CarMaker with the new Volvo XC90 vehicle model and performance of the controller are compared with more conventional lateral stability controllers and unassisted manoeuvres. While lateral control can be of benefit in some cases, it can hurt in others. When combined with speed control however, consistent reductions in risk of secondary collisions are seen and in cases involving large velocity ratios or long obstacles, large reductions are observed.

\section{INTRODUCTION}

According to NHTSA, over 15000 collisions with oncoming vehicles were reported in 2004 in the US alone and accounted for an economic cost of close to a billion USD (Yanagisawa et al. 2007). Such collisions are a distinct possibility when performing evasive steering manoeuvres to avoid obstacles as shown in figure 1 .
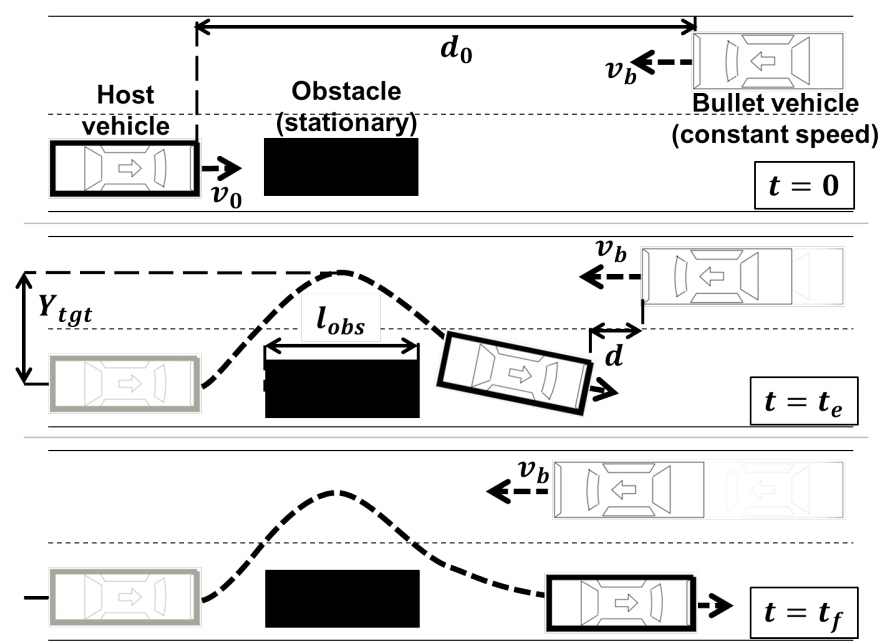

Figure 1: Obstacle avoidance with oncoming traffic
Evasive steering manoeuvres are necessary in many cases for obstacle avoidance (Brännström et al. 2010). While several assist systems for the same are already being developed (Dang et al. 2012), they ultimately rely on the driver to ensure a clear adjacent lane. As a result, while they may help the driver avoid an obstacle, if the adjacent lane turns out not to be free, the driver may then face a greater threat of collision with an oncoming vehicle. This paper outlines an approach to reduce this risk of secondary collisions by controlling the vehicle speed in the manoeuvre so as to minimize a combination of the time spent and the distance traveled over the manoeuvre.

The importance of controlling speed in this scenario can be illustrated by considering an extreme case wherein an aggressive lateral control intervention using differential braking during the manoeuvre can bring the host vehicle to a near halt in the wrong lane putting it squarely in the path of the oncoming vehicle (Arikere et al. 2015).

In the scenario considered in this paper, the driver initiates an evasive steering manoeuvre in order to avoid the obstacle. Awareness of the presence of an oncoming vehicle is not assumed for the driver. The 
vehicle is assumed to be able to detect the oncoming vehicle once the front edge of the longitudinal centre plane of the host vehicle clears the obstacle. This is in line with the industry practice of placing the radar and/or camera vision systems in the centre of the vehicle at the front. Due to the atypical and emergency nature of this manoeuvre, the driver steering intervention is expected to be inconsistent and unpredictable. The control system then attempts to reduce the risk of collision with said oncoming vehicle once the driver has started performing the steering manoeuvre. This task entails both lateral control to enhance vehicle response and stability and also speed control. The lateral control aspect is needed to ensure stability in the event of excessive or erratic steering interventions by a panicked driver. Additionally, it greatly increases the intensity of longitudinal interventions that can be performed without losing stability. The lateral control module used in this work is taken from Arikere et al. (2015). Note that this work considers only the vehicle dynamic control task in this manoeuvre and as such, other parts such as environmental perception and tactical decisions are working as required.

\section{SCENARIO VARIATIONS AND ACTUATORS}

As in Arikere et al. (2015), two variations of the scenario are considered (table 1) which represent the two extreme ends of the spectrum in terms of benefit of controlling the speed - one where it is expected to be of maximal benefit (Scenario A with large velocity ratio $\left(v_{b} / v_{0}\right)$ and long obstacle $\left.\left(l_{o b s}\right)\right)$ and another where it is expected to have minimal benefit (Scenario B with small velocity ratio and short obstacle). The risk of collision with the oncoming vehicle in these scenarios is measured using the distance margin $(d)$ which is the distance between the host vehicle and oncoming vehicle at the end of the manoeuvre. The end of the manoeuvre is defined as the instant when the host vehicle returns to the original lane after having passed the obstacle. Since steering interventions by a panicked driver in such an emergency situation tend to be inconsistent, different levels of steering severity are also considered for each scenario variation by changing the preview parameters of the driver model. The severity of the steering so achieved is measured using the steering effort metric $\left(E_{s t r}\right)$. In this scenario, the steering is assumed to be controlled by the driver (represented by the driver model). (see Arikere et al. (2015), Klomp et al. (2014) for details about the driver model).

Table 1: Manoeuvre parameters and their variations considered

\begin{tabular}{lccl}
\hline Parameters & Scenario A & Scenario B & Unit \\
\hline Host initial velocity $\left(v_{0}\right)$ & 60 & 120 & $\mathrm{~km} / \mathrm{h}$ \\
Bullet vehicle velocity $\left(v_{b}\right)$ & 120 & 60 & $\mathrm{~km} / \mathrm{h}$ \\
Obstacle length $\left(l_{\text {obs }}\right)$ & 15 & 3 & $\mathrm{~m}$ \\
Lateral displacement $\left(Y_{\text {tgt }}\right)$ & 1.5 & 1.5 & $\mathrm{~m}$ \\
\hline
\end{tabular}

Two driveline setups are considered in this analysis: a traditional internal combustion (IC) engine and an electric drive based all-wheel drive driveline. The IC engine driveline is assumed to be too slow to be able to supply drive torques to the wheels reliably and is hence not used for speed control. Hence with an IC engine driveline, the brakes are used to perform both lateral and speed control. The electric driveline on the other hand, is assumed to be quick enough and when available, is used in conjuction with differential braking to perform torque vectoring and also used for speed control.

\section{CONTROL DESIGN}

The wheel torque controller is split into three major modules namely, lateral control, speed control and the wheel torque allocator. The overall structure of the controller is shown in figure 2.

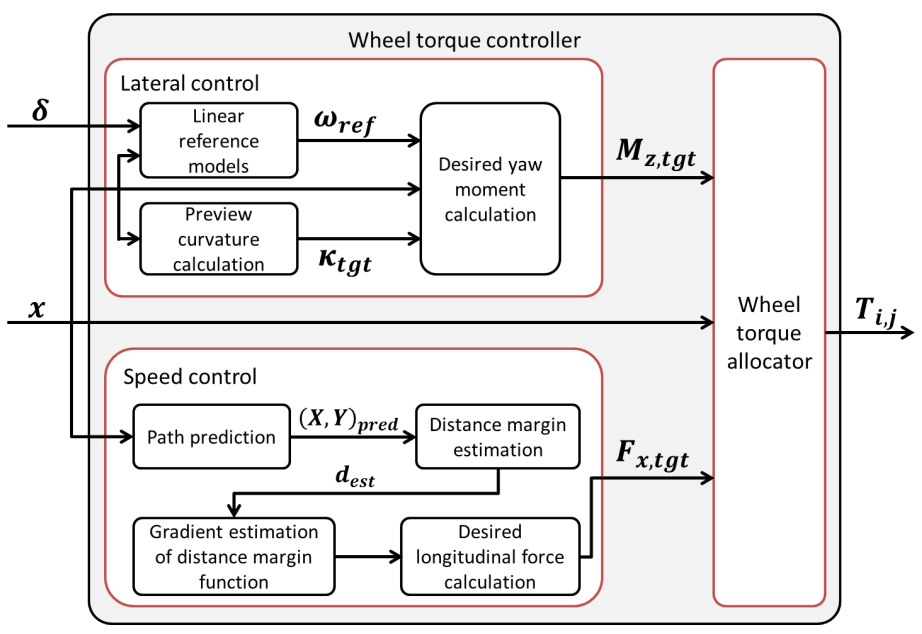

Figure 2: Wheel torque controller structure

\subsection{Lateral control}

The lateral control component consists of linear reference models to predict the yaw dynamics of the vehicle based on driver steering input, a preview curvature calculation part that determines the required curvature to avoid the obstacle or come back to the original lane and a desired yaw moment generator that outputs the yaw moment required for the vehicle to follow the target curvature. The lateral control component is taken from (Arikere, Lidberg, \& Olsson 2015) and is hence not described further in this work.

\subsection{Speed control}

The speed controller consists of four modules, one each for path prediction, distance margin estimation, gradient estimation of the distance margin function with respect to host vehicle speed and finally, the desired longitudinal force generator that increases the distance margin.

First, the path prediction module generates a simple path constructed of circular arcs and straight lines that 
the host vehicle is expected to follow. A point mass vehicle and constant vehicle speed are assumed while generating the path. These assumptions are warranted in this case since the steering intervention has a dominant effect on the vehicle path and the distance margin, and since it is being performed by the driver, is unknown to the controller. As such, simplyfing assumptions are necessary since more accurate predictions are not even possible.

As shown in figure 3 , the path consists of six nodes that fully determine the path. The point $\boldsymbol{P}_{\mathbf{0}}$ represents the current position of the host vehicle and the direction of the tangent at $\boldsymbol{P}_{\mathbf{0}}$ represents its heading angle. Apart from the segement $\boldsymbol{P}_{\mathbf{2}} \boldsymbol{P}_{\mathbf{3}}$, the remaining segments are made up of circular arcs with radius $R$ given by the friction limit assuming an on-limit manoeuvre.

$$
R=\frac{v_{x}^{2}}{\mu g}
$$

where, $v_{x}$ is the current vehicle speed and $\mu$ is the road friction which is assumed to be known.

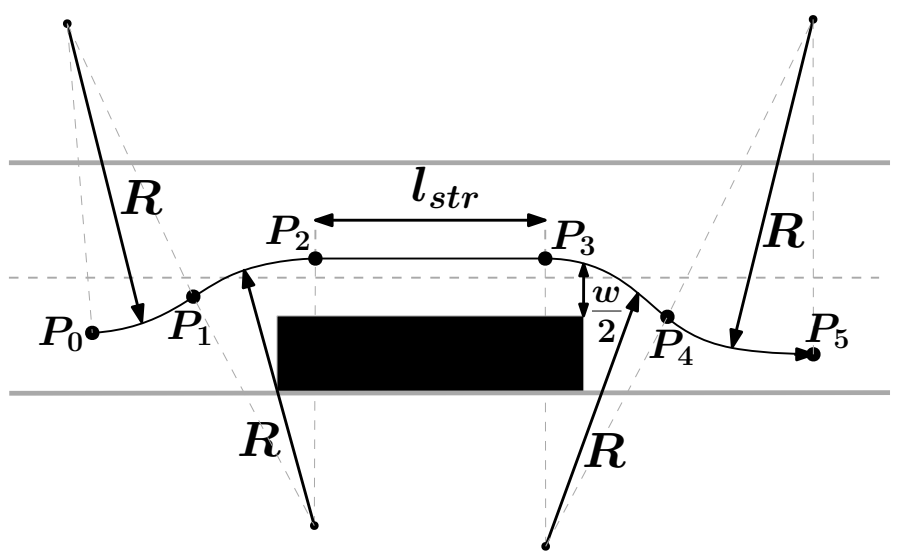

Figure 3: Predicted nominal path construction for speed control

The path is constructed assuming that the vehicle achieves the target lateral displacement as soon as possible and returns to original lane as soon as it clears the obstacle. Given these assumptions and a starting position $\boldsymbol{P}_{\mathbf{0}}$ and a heading angle $\psi_{0}$, the points $\boldsymbol{P}_{\mathbf{1}}$ and $\boldsymbol{P}_{2}$ are then determined. The point $\boldsymbol{P}_{1}$ is where the vehicle changes direction and starts turning right and $\boldsymbol{P}_{2}$ is when the vehicle has achieved the target lateral displacement and is travelling straight (zero heading angle).

$$
\begin{aligned}
& \psi_{1}=\arccos \left[\frac{1}{2 R}\left(Y_{0}-Y_{\text {tgt }}+R\left(1+\cos \psi_{0}\right)\right)\right] \\
& \psi_{2}=0 \\
& \boldsymbol{P}_{\mathbf{1}}=\boldsymbol{P}_{\mathbf{0}}+R\left(\sin \psi_{1}-\sin \psi_{0}, \cos \psi_{0}-\cos \psi_{1}\right) \\
& \boldsymbol{P}_{\mathbf{2}}=\boldsymbol{P}_{\mathbf{1}}+R\left(\sin \psi_{1}-\sin \psi_{2}, \cos \psi_{2}-\cos \psi_{1}\right)
\end{aligned}
$$

Next, since the length $l_{s t r}$ is unknown for the moment, the point $\boldsymbol{P}_{\mathbf{3}}$ is temporarily set equal to $\boldsymbol{P}_{\mathbf{2}}$ and the points $\boldsymbol{P}_{4}$ and $\boldsymbol{P}_{5}$ are provisionlly determined. Similar to points $\boldsymbol{P}_{\mathbf{1}}$ and $\boldsymbol{P}_{\mathbf{2}}, \boldsymbol{P}_{\mathbf{4}}$ is when the vehicle changes direction again and starts turning left and $\boldsymbol{P}_{\mathbf{5}}$ is when the vehicle is back at the starting $Y$ position $(=0)$ and is travelling straight again.

$$
\begin{aligned}
& \psi_{4}=-\arccos \left[\frac{1}{2 R}\left(R\left(1+\cos \psi_{3}\right)-Y_{3}\right)\right] \\
& \psi_{5}=0 \\
& \boldsymbol{P}_{\mathbf{4}}=\boldsymbol{P}_{\mathbf{3}}+R\left(\sin \psi_{3}-\sin \psi_{4}, \cos \psi_{4}-\cos \psi_{3}\right) \\
& \boldsymbol{P}_{\mathbf{5}}=\boldsymbol{P}_{\mathbf{4}}+R\left(\sin \psi_{5}-\sin \psi_{4}, \cos \psi_{4}-\cos \psi_{5}\right)
\end{aligned}
$$

Now, the straight line segment is added between $\boldsymbol{P}_{\mathbf{2}}$ and $\boldsymbol{P}_{\mathbf{3}}$ such that at the leading edge of the obstacle, the path is a distance $w / 2$ (half track width) away from the obstacle laterally. For this, first the point along the path where it just passes the obstacle has to be determined. This can happen either along $\boldsymbol{P}_{\mathbf{3}} \boldsymbol{P}_{\mathbf{4}}$ or along $\boldsymbol{P}_{\mathbf{4}} \boldsymbol{P}_{\mathbf{5}}$.

If $Y_{4}<\left(w_{o b s}+w / 2\right)$, the path crosses the leading edge of the obstacle along $\boldsymbol{P}_{\mathbf{3}} \boldsymbol{P}_{\mathbf{4}}$ :

$$
\psi_{o b s}=-\arccos \left[\frac{1}{R}\left(w_{o b s}+w / 2-Y_{3}+R \cos \psi_{3}\right)\right]
$$

$X_{o b s}=X_{3}+R\left(\sin \psi_{3}-\sin \psi_{o b s}\right)$

Else, the path crosses the leading edge of the obstacle along $\boldsymbol{P}_{4} \boldsymbol{P}_{5}$ :

$$
\psi_{\text {obs }}=-\arccos \left[\frac{1}{R}\left(Y_{4}-w_{o b s}-w / 2+R \cos \psi_{4}\right)\right]
$$

$X_{o b s}=X_{4}+R\left(\sin \psi_{o b s}-\sin \psi_{4}\right)$

Given the $X$ position of the leading edge of the obstacle as $X_{o b s, f r}$, the length of the straight line segment to be added between $\boldsymbol{P}_{\mathbf{2}}$ and $\boldsymbol{P}_{\mathbf{3}}$ can be calculated as follows:

$$
l_{s t r}=X_{o b s, f r}-X_{o b s}
$$

With this, the point $\boldsymbol{P}_{\mathbf{3}}$ can be fully determined and the points $\boldsymbol{P}_{\mathbf{4}}$ and $\boldsymbol{P}_{\mathbf{5}}$ are recalculated using the new $\boldsymbol{P}_{\boldsymbol{3}}$ with equations (8) and (9).

$$
\boldsymbol{P}_{\mathbf{3}}=\boldsymbol{P}_{\mathbf{2}}+\left(l_{s t r}, 0\right)
$$

The path is continously reconstructed over the course of the manoeuvre in order to account for the deviations in the actual path that invariably occur due to the driver steering. Note that the equations given above are for the case when the vehicle is in the early stages of the manoeuvre. As such, many of the equations yield invalid values when the vehicle is further 
into the manoeuvre. For instance, when the vehicle is halfway into passing the obstacle, the estimate $\psi_{1}$ and $\psi_{2}$ will become invalid. The estimates are therefore checked every iteration and when they become invalid, it indicates that the vehicle is already past the point being determined. These estimates and points are then set equal to the current vehicle position and heading angle and the remaining points are estimated as usual. Shown in figure 4 is the evolution of the predicted vehicle path over the course of the manoeuvre and the actual vehicle path.

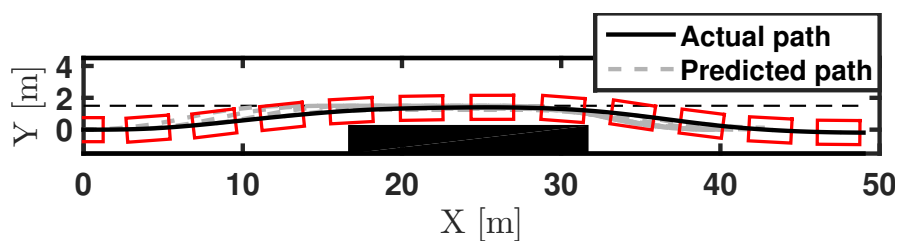

Figure 4: Predicted and actual vehicle path

Once the path is fully determined, the distance margin is then estimated.

$d=d_{0}-\underbrace{\left(d_{h, x}+\frac{d_{h}}{v_{x}} v_{b}\right)}_{d_{t r}}$

where, $d_{0}$ is the current distance between the host vehicle and the bullet vehicle, $d_{h}$ is the length of the path (distance travelled by the host vehicle) and $d_{h, x}$ is the length of the predicted path along the $\boldsymbol{X}$ axis. The distance travelled by the two vehicles over the course of the manoeuvre is indicated as $d_{t r}$. Both the host and the bullet vehicle are assumed to travel at constant speeds in this estimation. The lengths $d_{h}$ and $d_{h, x}$ are computed from the path parameters as follows:

$$
\begin{aligned}
d_{h} & =l_{s t r}+\Sigma_{i=0}^{4}(-1)^{i} R\left(\psi_{i+1}-\psi_{i}\right) \\
d_{h, x} & =l_{s t r}+\sum_{i=0}^{4}(-1)^{i} R\left(\sin \psi_{i+1}-\sin \psi_{i}\right)
\end{aligned}
$$

The distance margin, $d$ here is the objective that we intend to maximise by controlling the host vehicle speed. Hence the derivative of $d$ with respect to the host vehicle speed, $v_{x}$ should give an indication whether we need to increase or decrease vehicle speed to reduce the risk of collision with the oncoming vehicle. Since $d_{0}$ is a constant here, the expression for the derivative can be simplified as follows:

$\frac{\mathrm{d} d}{\mathrm{~d} v_{x}}=-\frac{\mathrm{d} d_{t r}\left(v_{x}\right)}{\mathrm{d} v_{x}}=J_{v_{x}}$

The resulting slope of the objective $\left(J_{v_{x}}\right)$ was then simply multiplied by a tunable gain factor to yeild a longitudinal force target $\left(F_{x, t g t}\right)$. For safety, the resulting $F_{x, t g t}$ was also limited to a certain pre-determined bound to prevent saturating the tyres.

$F_{x, t g t}=\min \left(\max \left(K_{\text {long }} J_{v_{x}},-F_{x, \max }\right), F_{x, \max }\right)$
Other more sophisticated methods such as running an optimisation within the controller or taking a Newton Step, etc to determine the optimal $F_{x, t g t}$ were tried out, however, due to non-smooth nature of the objective function, they resulted often in rapid and sudden interventions that sometimes made the situation worse. Furthermore, since the second derivative of the objective is discontinous, the Newton Step method could not be reliably applied. It was found that the simple method of multiplying the slope with a constant gain resulted in more robust behaviour, albeit in many cases, performed worse than the more sophisticated methods.

\subsection{Wheel torque allocator}

Finally, the control allocation block takes the longitudinal force target $\left(F_{x, t g t}\right)$, the yaw moment target $\left(M_{z, t g t}\right)$ and the vehicle states $(x)$ and configuration as inputs and generates target wheel torques to be applied on the four wheels.

Once again, the wheel torque control module is largely taken from Arikere et al. (2015) with some minor changes to take into account the new input of $F_{x, t g t}$ as well. Mainly, the control allocator takes into account the capability of the actuator setup (whether it has electric propulsion and on which axle it has this capability), whether the vehicle is understeering or oversteering and the load distribution between the axles due to the vehicle centre of gravity and longitudinal acceleration, and tries to distribute the wheel torques accordingly to achieve the global force targets. The reader is directed to the section Wheel force controller in Arikere et al. (2015) for more details.

\section{INCREASED DISTANCE MARGIN WITH SPEED CONTROL}

Simulations are preformed with the controllers designed above in IPG CarMaker. A new Volvo XC90 vehicle model is used in the simulations (figure 5).

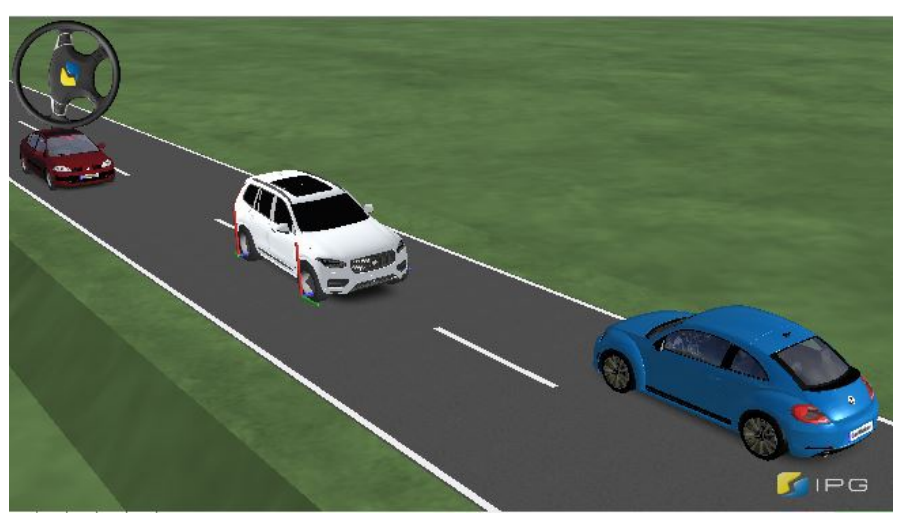

Figure 5: Simulation setup in CarMaker

Figure 6 shows the performance of speed control when used with an IC engine driveline. As can be seen, using speed control provides consistently better performance. In scenario A, where maintaining speed is of 


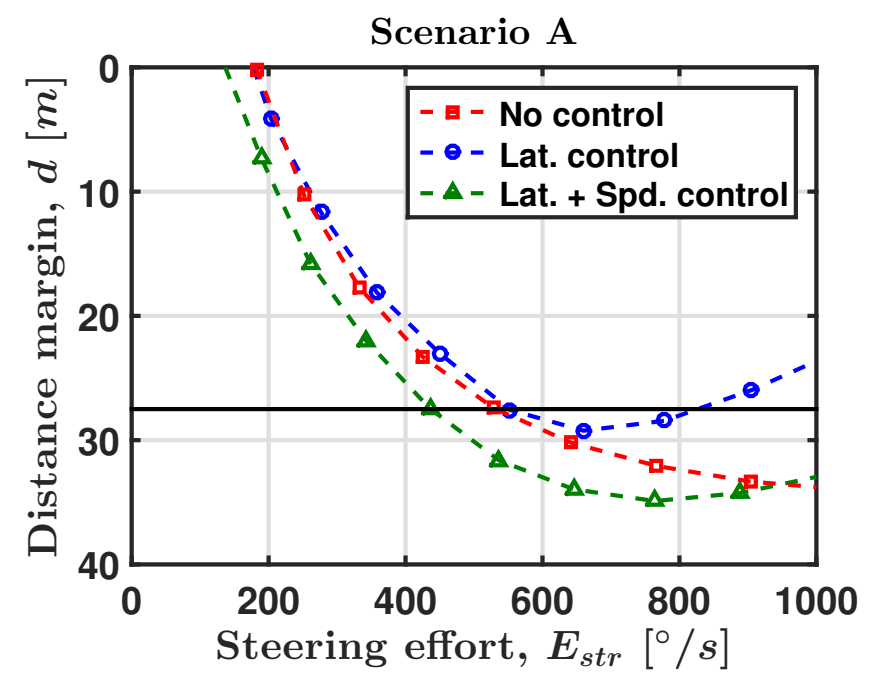

(a)

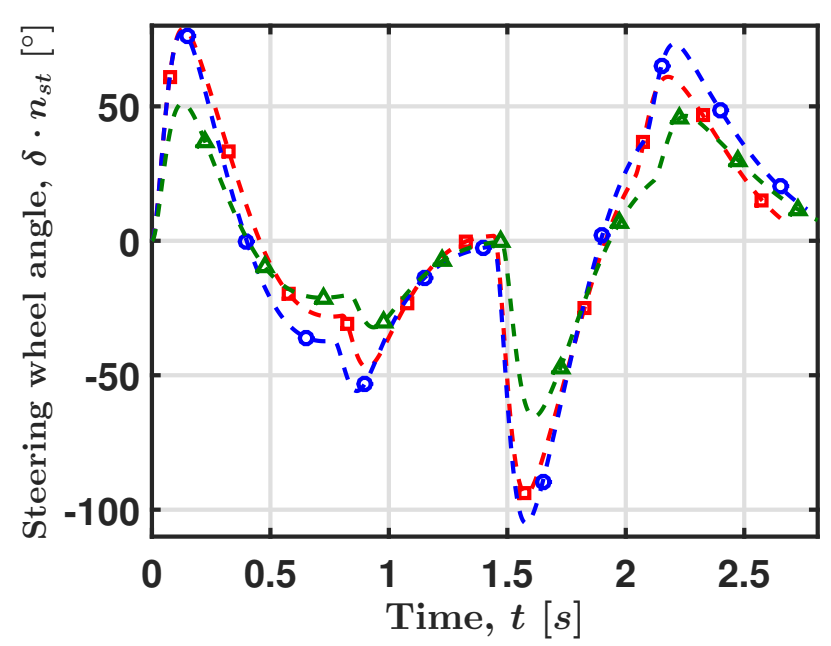

(c)

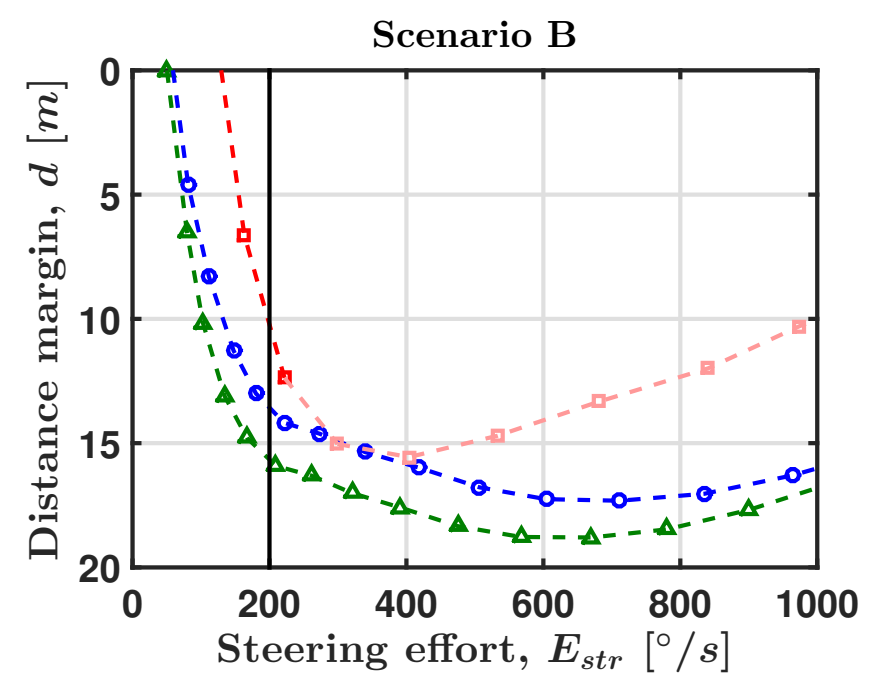

(b)

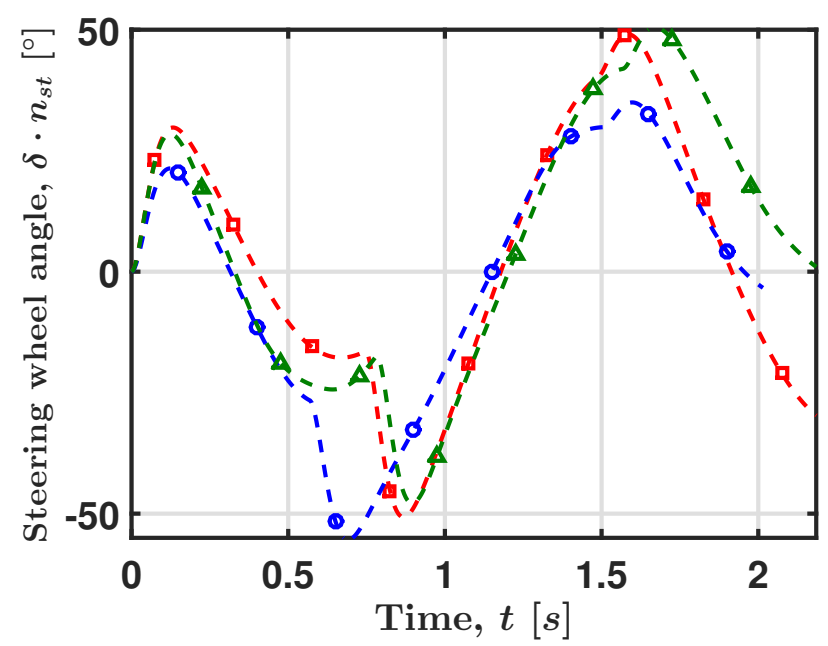

(d)

Figure 6: The pareto fronts for steering effort vs distance margin for scenario A and B using the speed controllers for an IC engine based driveline are shown in (a) and (b) respectively. Lateral control is performed using differential braking. Steering wheel angle profiles corresponding to the marked distance margin level (horizontal line) in (a) are shown in (c) and those corresponding to the marked steering intrusiveness level (vertical line) in (b) are shown in (d).

benefit, it can be seen that differential braking alone can deteriotate performance in many cases. However, with speed control added, the system consistently performs better. In scenario B on the other hand where reducing speed is of benefit, differential braking works well on its own. As a result, adding speed control does not provide much benefit, but still manages to match the performance in the worst case and improve it slightly in some cases. Note also that the lighter shades of the plot for the "No control" case indicates that the vehicle fails to finish the manoeuvre in a stable fashion.

In figures 6(c) and 6(d), the steering profiles corresponding to the intercepts of the horizontal and vertical line in figures 6(a) and 6(b) are shown respectively. It can be seen that, in case of figure 6(c), although the steering profiles look similar, the peak steering rate and the steering amplitudes are significantly smaller for the case with speed control. On the other hand, in figure $6(\mathrm{~d})$, it can be seen that even though the profiles look similar, the peak steering rates are nearly the same for all the cases. These observations match with what we expect from the steering profiles based on their steering intrusiveness values.

Shown in figure 7 is the performance of speed control when used with an electric driveline. Here, the electric driveline and the differential brakes are used together to achieve torque vectoring which is used for lateral control. Note that for safety, acceleration is not permitted and at best, the controller only maintains speed. In scenario A here, the use of torque vectoring benefits greatly as it performs lateral control without slowing the vehicle down. As a result, adding speed control here does not help much. In scenario B however, where reducing speed is of benefit, while torque vectoring alone is of help, adding speed control significantly increases the distance margin as it also slows the vehicle down. This is because when lateral control alone is performed with torque vectoring, the vehicle does not slow down which is detrimental for safety in this case. When speed control is added, the vehicle is slowed down in addition to performing lateral control 


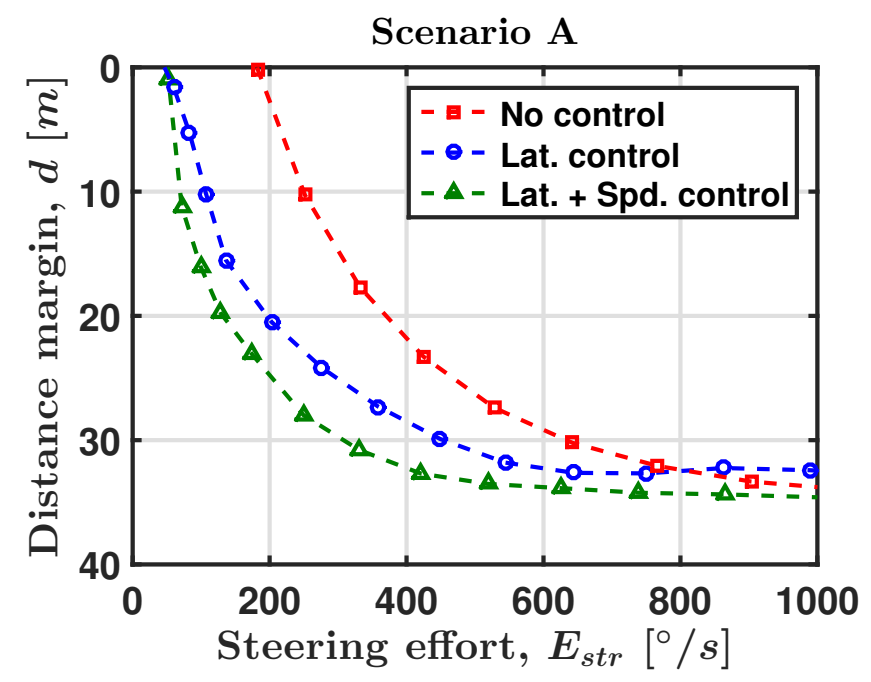

(a)

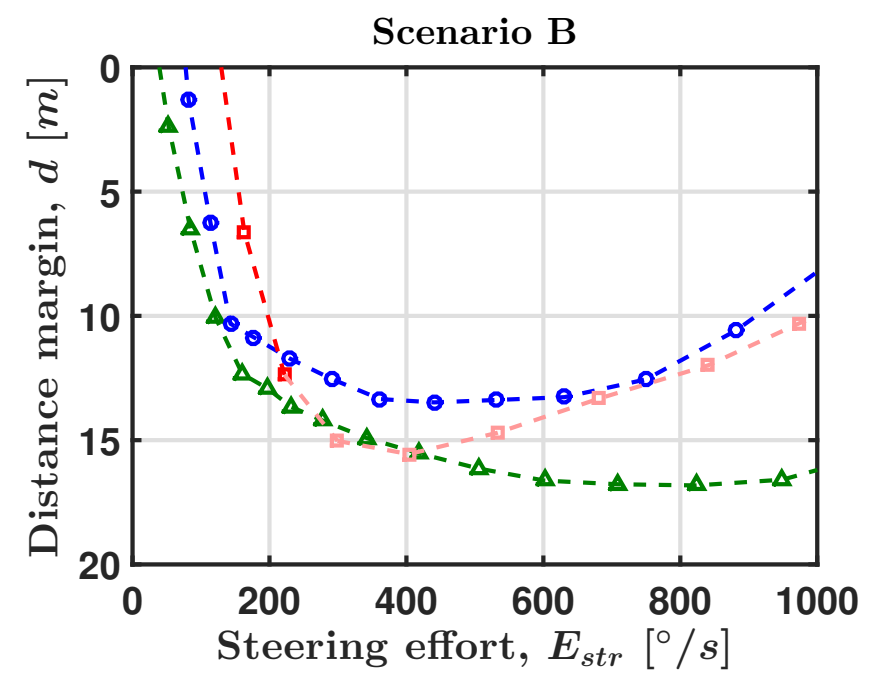

(b)

Figure 7: The pareto fronts for steering effort vs distance margin for scenario A and B using the speed controllers for an electrified driveline are shown in (a) and (b) respectively. Lateral control is performed using torque vectoring.

which improves safety.

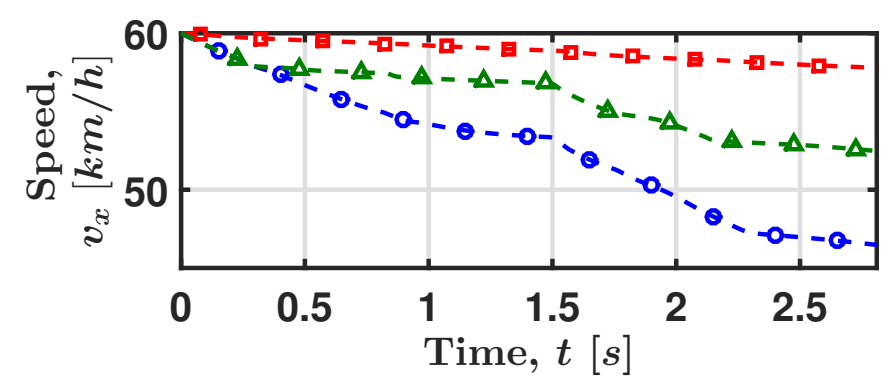

Figure 8: Speed profiles for the cases in figure 6(a) where $E_{\text {str }} \approx$ $400^{\circ} / \mathrm{s}$

Figure 8 shows the speed profiles for the cases in figure 6(a) with steering intrusiveness $\left(E_{s t r}\right)$ of $400^{\circ} / \mathrm{s}$. As can be seen, when lateral control alone is performed, the vehicle is stabilised but at the cost of speed. Whereas when speed control is added, it manages to achieve a better tradeoff between lateral control and speed control which leads to a higher distance margin.

\section{CONCLUSIONS}

Simulations using the outlined strategy to control speed showed that large reductions in oncoming vehicle collision risk can be achieved in scenarios involving large velocity ratios and long obstacles. Smaller but consistent reductions in collision risk with oncoming vehicles can also be achieved in other scenarios.

Noticable increases in distance margin are achieved even when used with traditional IC engine drivetrains. However, for large and consistent improvements in distance margin over differential braking, an electrified drivetrain is needed. Large improvements are seen with electric drivelines even when only lateral control (torque vectoring) is performed. This benefit is amplified even further when speed control is added.

Since consistent improvements in distance margin are seen irrespective of the scenario or the type of drivetrain, speed control can therefore be seen as an effective option for mitigating the risk of collision with oncoming vehicles when evasive steering manoeuvres are performed for obstacle avoidance.

\section{Acknowledgements}

This project was partly funded by the Fordonsstrategisk Forskning och Innovation (FFI) program of VINNOVA and the vehicle models used in simulations were provided by Volvo Car Corporation. Their support is gratefully acknowledged.

The support of IPG in supplying an academic version of CarMaker for use in this research is also gratefully acknowledged.

\section{REFERENCES}

Arikere, A., M. Lidberg, \& G. Olsson (2015, August). The trade-off between distance margin and steering effort in obstacle avoidance manoeuvres with oncoming traffic. In Proceedings of the 24th International Symposium on Dynamics of Vehicles on Roads and Tracks, Graz, Austria.

Brännström, M., E. Coelingh, \& J. Sjöberg (2010). ModelBased Threat Assessment for Avoiding Arbitrary Vehicle Collisions. IEEE Transactions on Intelligent Transportation Systems 11(3), 658-669.

Dang, D. T., D. J. Desens, D. U. Franke, P. D. D. Gavrila, L. Schäfers, \& D. W. Ziegler (2012). Steering and Evasion Assist. In A. Eskandarian (Ed.), Handbook of Intelligent Vehicles, pp. 759-782. Springer London. DOI: 10.1007/978-0-85729-085-4_29.

Klomp, M., K. Olsson, \& C. Sandberg (2014, January). Nonlinear steering control for limit handling conditions using preview path curvature. International Journal of Vehicle Autonomous Systems 12(3), 266-283.

Yanagisawa, M., J. D. Smith, \& W. G. Najm (2007, April). Pre-Crash Scenario Typology for Crash Avoidance Research. Technical report DOT HS 810 767, NHTSA. 